\title{
Physical Properties of Laterally Fluorinated Isothiocyanato Phenyl-tolane Single Liquid Crystals Components and Mixtures
}

\author{
Sebastian Gauza ${ }^{1}$, Amanda Parish ${ }^{1}$, Shin-Tson Wu ${ }^{1}$, \\ Anna Spadło ${ }^{2}$, and Roman Dabrowski ${ }^{2}$ \\ ${ }^{1}$ College of Optics and Photonics, University of Central Florida, Orlando, \\ Florida, USA \\ ${ }^{2}$ Institute of Chemistry, Military University of Technology, Warsaw, \\ Poland
}

We have designed, synthesized, and evaluated the physical properties of some high birefringence $(\Delta n)$ isothiocyanato phenyl-tolane liquid crystals. These compounds exhibit a $\Delta n \simeq 0.48-0.52$ at room temperature and wavelength $\lambda=633 \mathrm{~nm}$. Laterally substituted short alkyl chains and fluorine atom both eliminate smectic phase formation and lower the melting temperature. Both the moderate melting temperature and very high clearing temperature make those compounds attractive for eutectic mixture formulation. Examples of the mixtures containing the described components are presented.

Keywords: high birefringence; isothiocyanato phenyl-tolanes; liquid crystals

\section{INTRODUCTION}

High birefringence liquid crystal (LC) materials are important for laser beam steering [1], tunable-focus lens [2], reflective display [3], infrared dynamic scene projector [4], and telecom variable optical attenuator [5] applications. Fast response time is particularly important for color-sequential liquid crystal displays (LCDs) using a blinking backlight [6] or primary color (RGB) light emitting diodes (LEDs) [7,8]. For these devices, high $\Delta \mathrm{n}$ improves response time

This work is supported by DARPA Bio-Optics Synthetic Systems program under Contract No. W911NF04C0048, and NATO Programme Security Through Science, Collaborative Linkage Grant No. CBP.EAP.CLG 981323.

Address correspondence to Shin-Tson Wu, College of Optics \& Photonics, 4000 Central Florida Blvd, PO Box 162700, Orlando, FL 32816-2700, USA. E-mail: swu@mail.ucf.edu 
through cell gap $(d)$ reduction. This advantage is especially important for laser beam steering and variable optical attenuators as these devices are normally operated in the near infrared region $(\lambda=1.55 \mu \mathrm{m})$. In the long wavelength region, the LC optical path difference $(\mathrm{d} \Delta \mathrm{n})$ should increase in order to achieve the required phase change. High birefringence helps to reduce the cell gap in order to maintain fast response time [9-11].

To increase birefringence, linearly conjugated molecules are the preferred candidates $[12,13]$. Conjugation length can be extended either by multiple bonds or by unsaturated rings in the rigid core [14]. The three most important problems associated with highly conjugated LC compounds are high melting temperature, increased viscosity, and reduced UV stability. Eutectic mixtures can be used to overcome high melting temperature. The increased viscosity is inherent to all the highly conjugated compounds. Isothiocyanato (NCS) compounds are less viscous than the cyano (CN) ones, but the consequence of utilizing NCS compounds is that they tend to exhibit smectic phases [15]. Due to increased absorption at UV wavelengths, these high birefringence compounds need to be protected from direct UV exposure but in general are suitable for infrared applications [16].

Several high birefringence molecular structures, such as cyano and NCS tolanes [17,18], diphenyl-diacetylene [19,20], bistolane [21,22], naphthalene tolanes [23,24], and thiophenyl-diacetylene [25-27] have been studied. Recently several highly conjugated compounds were synthesized and their properties were investigated [28-31]. The birefringence of these compounds is in the 0.4-0.8 range. However, some super high birefringence compounds [31] tend to have a large viscosity and both their chemical and photo-stabilities are a concern.

In this paper, we present several isothiocyanato phenyl-tolane compounds with extrapolated birefringence $\sim 0.48-0.52$ at $\lambda=633 \mathrm{~nm}$ and $\mathrm{T}=23^{\circ} \mathrm{C}$. To lower the melting temperature, we tried different lateral fluorinations.

\section{EXPERIMENTAL}

Several measurement techniques were used to measure the physical properties of the single compounds and mixtures. Differential Scanning Calorimetry (DSC, TA Instrument Model Q-100) was used to determine the phase transition temperatures. Results were obtained from $3-6 \mathrm{mg}$ samples in the heating and cooling cycles with rate $2{ }^{\circ} \mathrm{C} / \mathrm{min}$ for both cycles.The electro-optic properties of the LC compounds and mixtures were measured using $5-\mu \mathrm{m}$ path length cells with ITO (indium-tin-oxide) electrodes coated in the inner sides of 
the glass substrates. A thin polyimide layer was overcoated on ITO and buffed in anti-parallel directions to produce homogeneous LC alignment with a small pretilt angle $\left(\sim 2^{\circ}\right)$. A linearly polarized He-Ne laser with $\lambda=633 \mathrm{~nm}$ was used as the light source for the electro-optic measurements. Experimental setup and measurement technique were the same as those reported in [32].

\section{RESULTS AND DISCUSSIONS}

\subsection{Single Compounds}

Table 1 lists the molecular structures and phase transition temperatures of eight isothiocyanato phenyl-tolane LCs investigated. Even with lateral substitution(s), the melting temperatures of these compounds are relatively high. The difluoro substituted isothiocyanato-tolane compounds, $\mathbf{1}$ and $\mathbf{2}$, exhibit a wide nematic range with clearing point temperatures exceeding $200^{\circ} \mathrm{C}$. The propyl homologue (Compound 1) shows a melting transition at $122^{\circ} \mathrm{C}$ which is relatively high. The pentyl homologue (Compound 2) shows a much lower melting point temperature of $55^{\circ} \mathrm{C}$, but its smectic to nematic transition occurs at $119^{\circ} \mathrm{C}$. The heat fusion enthalpy $(\Delta \mathrm{H})$ is significantly different for the $\mathrm{C} 3$ and C5 homologues. Compound 1, having a shorter alkyl chain, shows $\Delta \mathrm{H} \sim 6.3 \mathrm{kcal} / \mathrm{mol}$ which is $\sim 2 \mathrm{X}$ higher than that of Compound 2, which has a five carbons alkyl chain. A different lateral fluorination scheme was utilized for the second homologue series (Compounds 3, 4, and 5) investigated. This triple fluorination scheme has a fluorine substitution in the second and third positions of the middle phenyl ring while the last phenyl ring has a single fluorine substitution at the position adjacent to the NCS terminal group. The measured melting point temperature for these compounds is significantly lower than that observed for difluorinated Compound 1. Only the ethyl homologue does not exhibit a smectic phase. The pentyl homologue in this series shows an exceptionally low heat fusion enthalpy $\Delta \mathrm{H} \sim 3.4 \mathrm{kcal} / \mathrm{mol}$. Although the melting temperatures for Compounds $2,3, \mathbf{4}$, and $\mathbf{5}$ are relatively low, the smectic phase present in the propyl and pentyl homologues decreases their attractiveness for application as a nematic material. The positions of the laterally substituted fluorine in Compound $\mathbf{6}$ are different from the trifluorinated phenyl-tolanes already discussed. The middle phenyl ring has a fluoro group at the second position while the last phenyl linked with the terminal NCS group is fluorinated in the $(3,5)$ positions, similar to Compound $\mathbf{1}$ and $\mathbf{2}$. Comparing the propyl homologues (Compounds $\mathbf{1}$ and $\mathbf{6}$ ) shows that additional fluorine substituted at the middle ring 
TABLE 1 Molecular Structures and Phase Transition Temperatures of Five Phenyl-Tolane Isothiocyanates

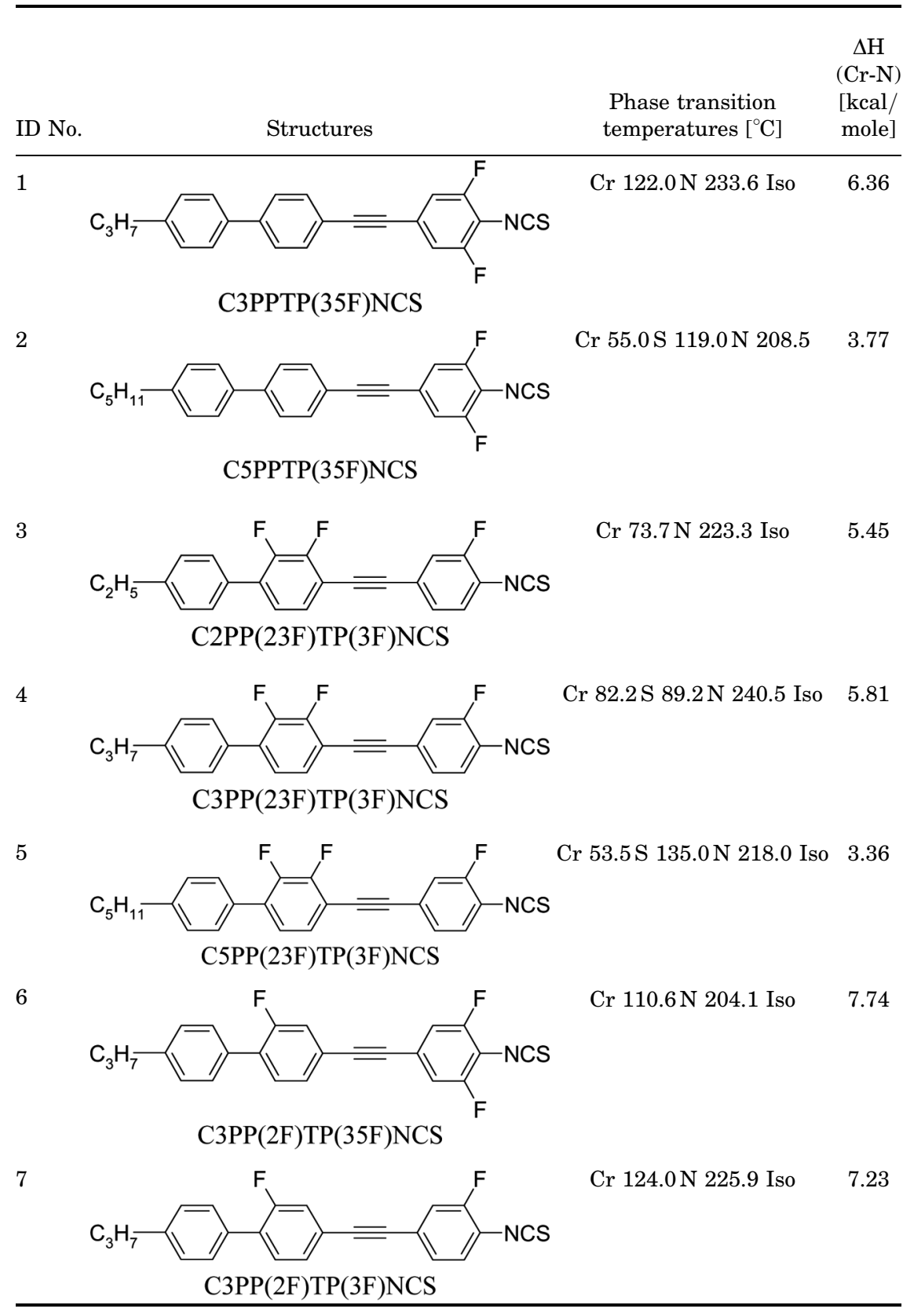


TABLE 1 Continued

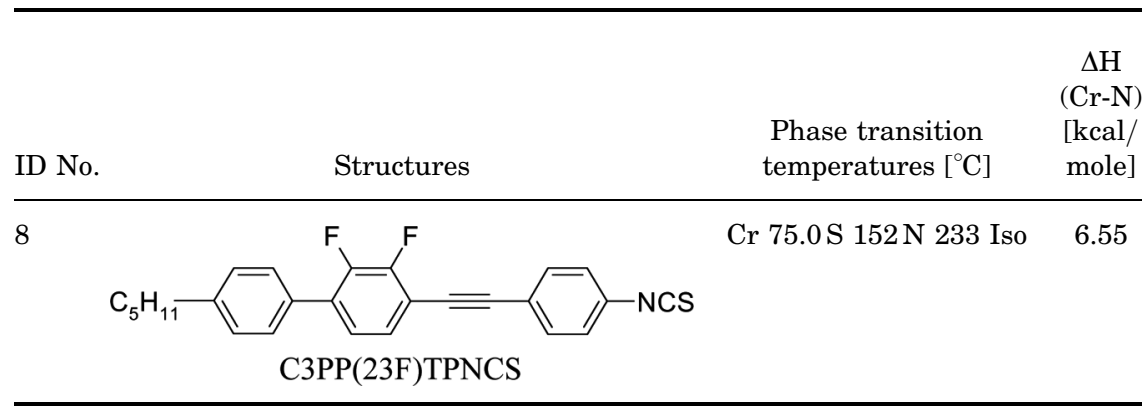

slightly decreases the melting temperature. Comparing the melting temperatures of Compounds $\mathbf{1}$ and $\mathbf{7}$ as well as $\mathbf{2}$ and $\mathbf{8}$ shows that fluorination of the phenyl ring adjacent to the NCS group results in the best mesomorphic properties of the difluorinated series considered.

Most device applications are performed at room temperature $\left(\sim 23^{\circ} \mathrm{C}\right)$. Thus, the physical properties of these high melting compounds at room temperature are of interest. Two methods are commonly employed to extrapolate the electro-optical properties of these single compounds at room temperature: 1 ) preparation of a guest-host system, and 2) parameter-fitting and extrapolation from elevated temperature results. In the first method, about $10 \mathrm{wt} \%$ of the guest compound to be investigated is dissolved in a room temperature LC host mixture. By knowing the birefringence of the host mixture, the $\Delta n$ of the guest compound at room temperature can be estimated according to the following equation:

$$
(\Delta n)_{g h}=x(\Delta n)_{g}+(1-x)(\Delta n)_{h},
$$

where $g h, g$, and $h$ denote the birefringence of the guest-host system, guest compound, and host mixture, respectively. In the second method, we measure the properties of the compound at several temperatures in its nematic phase, fit the experimental data to theoretical data generated by physicochemical models, and finally extrapolate the results to room temperature. In this study, we focused on the birefringence $(\Delta n)$, visco-elastic coefficient $\left(\gamma_{1} / K_{11}\right)$ and the "Figure-ofMerit" which is defined as [33]:

$$
F_{O M}=K_{11} \Delta n^{2} \gamma_{1}
$$

where $K_{11}$ is the elastic constant and $\gamma_{1}$ the rotational viscosity. The temperature dependent birefringence of an $\mathrm{LC}$ can be described as follows:

$$
\Delta n=\Delta n_{o}\left(1-T / T_{c}\right)^{\beta},
$$


where the fitting parameters are $\Delta n_{o}$, the birefringence at $\mathrm{T}=0^{\circ} \mathrm{K}$, and $\beta$, a material constant, and $T_{c}$ is the clearing temperature of the LC. By fitting the experimental data using Eq. (3), we can obtain $\Delta n_{o}$ and $\beta$. Once these two parameters are determined, the birefringence of the LC at room temperature can be extrapolated. Similarly we fit FoM with following equation [33]:

$$
F_{O} M=a(\Delta n)^{2}\left(1-\frac{T}{T_{c}}\right)^{3 \beta} \exp \left(\frac{-E}{\kappa T}\right)
$$

where $a$ is a fitting parameter. The extrapolated visco-elastic coefficient at room temperature can be calculated from Eq. (2). In this study, we chose to use the fitting and extrapolation method instead of guest-host method to determine compound properties at room temperature.

Figure 1a shows the temperature dependent birefringence of selected di- and tri-fluoro phenyl-tolanes. Among presented compounds, difluorinated Compound $\mathbf{1}$ has the highest birefringence, whereas the least attractive is Compound $\mathbf{6}$ based on this comparison. From Eq. (4) it is shown that the birefringence of an LC strongly depends on the clearing point temperature.

A longer alkyl chain slightly decreases the birefringence because of the lower molecular packing density. This effect is shown in Figure $1 \mathrm{~b}$ thru the comparison of Compounds 3, 4, and 5. Regarding to the two trifluoro substitutions, from Figure 1, Compounds 4 has higher birefringence than Compound 6. By measuring the free relaxation time of a homogeneous cell, we are able to calculate the visco-elastic coefficient of the LC compounds [34]. Results are depicted in Figure 2a. We found that Compounds 6 and 7 show the highest visco-elastic coefficient among the investigated LC structures. Compound 4, trifluorinated at different positions of the rigid core phenyl rings, shows a moderate visco-elastic coefficient. A low value of $\gamma_{1} / K_{11}$ was measured for the double fluorinated Compound 8 . Even with a long, five carbons alkyl chain, Compound $\mathbf{8}$ shows only slightly higher visco-elastic coefficient than Compound 1. Considering the difluorinated compounds with different positions of lateral fluorination, we observe the following sequence of $\gamma_{1} / K_{11}$ values:

\section{Compound $\mathbf{1}<$ Compound $8<<$ Compound $\mathbf{7}$}

When the $\gamma_{1} / K_{11}$ coefficient is compared for Compounds $\mathbf{3}, \mathbf{4}$, and $\mathbf{5}$, which share a common rigid core, the pentyl homologue (Compound 5) exhibits the lowest value. This result is similar to the comparison of double fluorinated compounds, for which the pentyl homologue 


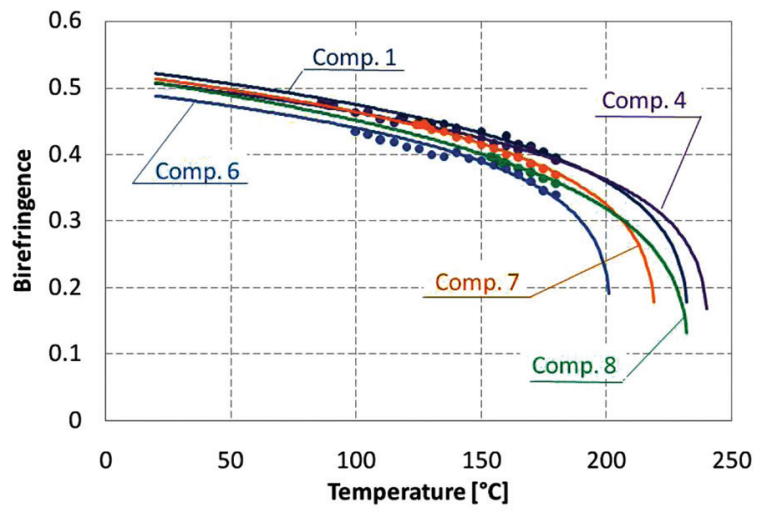

(a)

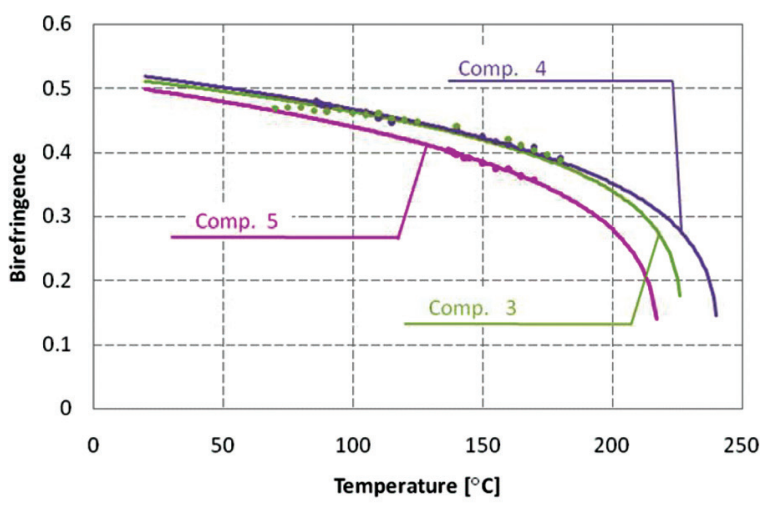

(b)

FIGURE 1 Temperature dependent birefringence of (a) short alkyl chain NCS-phenyltolanes and (b) different alkyl chain of the same rigid core homologues.

(Compound 8) also demonstrates the lowest $\gamma_{1} / K_{11}$ value. Surprisingly the ethyl homologue (Compound 3) exhibits a slightly higher viscoelastic coefficient than the other two homologues with a longer alkyl chain length, as shown in Figure 2b.

We would like to focus on the Figure-of-Merit (FoM) as the most important comparison. Figure $3 \mathrm{a}$ and $3 \mathrm{~b}$ shows the temperature dependent FoM of di- and tri-fluorinated phenyl-tolanes. Among all the compounds investigated, the difluorinated Compound 1 shows the highest FoM which is $180 \mu \mathrm{m}^{2} / \mathrm{s}$ at $160-170^{\circ} \mathrm{C}$. Conversely, the trifluorinated Compound 6 exhibits the lowest FoM (barely reaching 


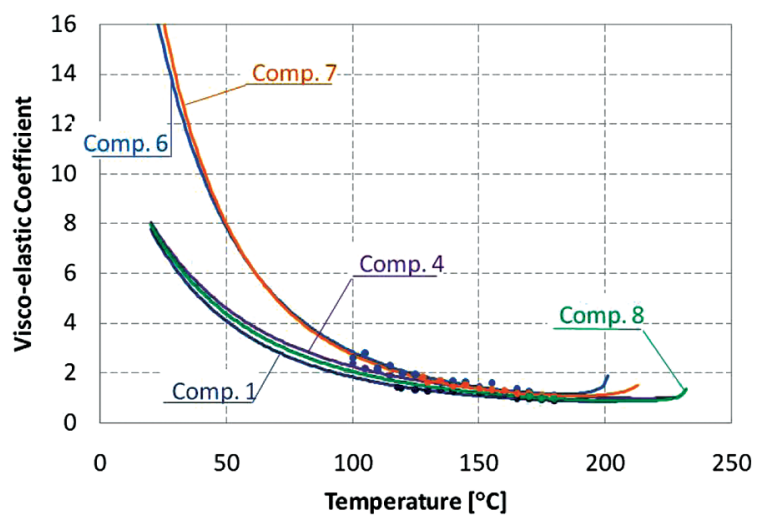

(a)

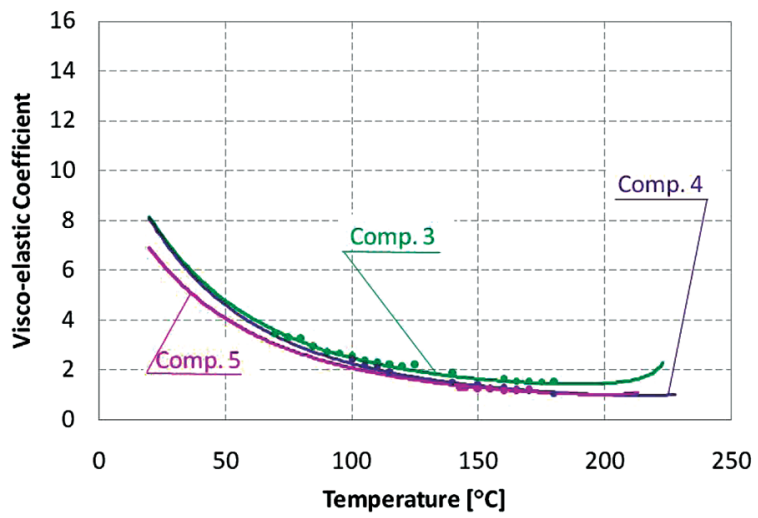

(b)

FIGURE 2 Temperature dependent visco-elastic coefficient of (a) short alkyl chain NCS-phenyltolanes and (b) different alkyl chain of the same rigid core homologues.

$100 \mu \mathrm{m}^{2} / \mathrm{s}$ at its optimal temperature) because it has the lowest birefringence and highest visco-elastic coefficient. From Table 1, Compound 4 is different from Compound $\mathbf{6}$ only in the positions of lateral fluorination, but their mesomorphic properties and electrooptical performance are quite different. Compound 6, with two fluoro substitutions adjacent to the NCS terminal group, has a high melting point and low clearing point and therefore has a much narrower nematic range as compared to Compound 4. The low $\mathrm{T}_{c}$ of Compound 6 negatively affects its birefringence and, moreover, its visco-elastic 


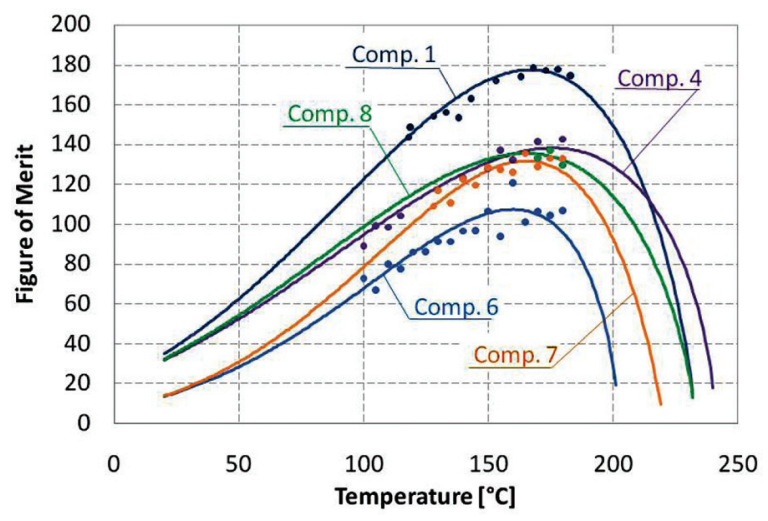

(a)

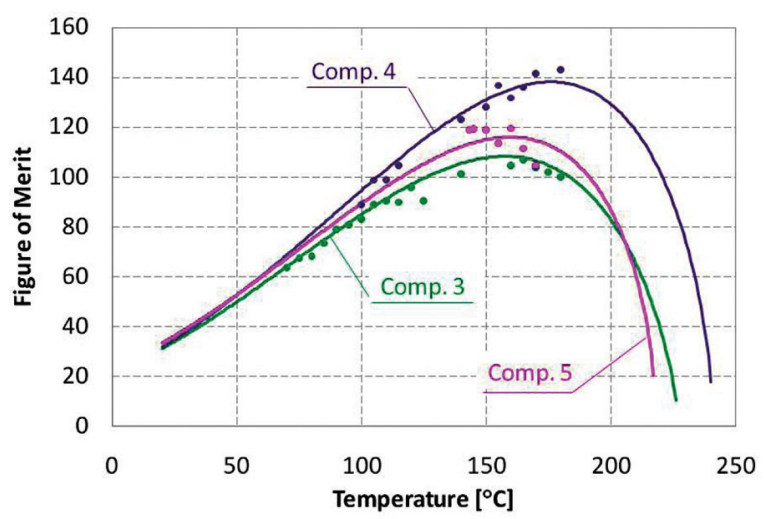

(b)

FIGURE 3 Temperature dependent figure of merit of (a) short alkyl chain NCS-phenyltolanes and (b) different alkyl chain of the same rigid core homologues.

coefficient is relatively high. As a result, its FoM is low. In contrast, Compound 4 demonstrates a respectable $\mathrm{FoM}$ of $140 \mu \mathrm{m}^{2} / \mathrm{s}$ at the optimal temperature, which is slightly higher than that of the difluorinated Compounds $\mathbf{7}$ and $\mathbf{8}$. For the Compounds 4, 6, 7, and $\mathbf{8}$, we find that there is no significant difference in FoM between the di- and tri-fluoro substitutions. Instead, the critical issue is the position at which the fluorine is substituted. Two compounds (6 and 7 ) with a single fluorination in the middle phenyl ring show a severe increase in visco-elastic coefficient which makes them less attractive from the point of view of fast response time. 
TABLE 2 Phase Transition Temperatures and Compositions of the Selected Doped Systems

\begin{tabular}{lcc}
\hline Doped system & Wt \% ratio (host:guest) & Phase transition temperatures $\left[{ }^{\circ} \mathrm{C}\right]$ \\
\hline UCF-D10 & $90: 10$ & $\mathrm{Cr} 8.9 \mathrm{~N} 124.7$ Iso \\
UCF-D20 & $80: 20$ & $\mathrm{Cr} 7.3 \mathrm{~S} 25.6 \mathrm{~N} 128.8$ Iso \\
UCF-D30 & $70: 30$ & $\mathrm{Cr} 12.7 \mathrm{~S} \mathrm{32.4} \mathrm{N} \mathrm{137.6} \mathrm{Iso}$ \\
UCF-D50 & $50: 50$ & $\mathrm{Cr} 29.1 \mathrm{~S} 62.7 \mathrm{~N} \mathrm{160.1} \mathrm{Iso}$ \\
\hline
\end{tabular}

\subsection{High Birefringence Mixtures}

Several mixtures were composed using different concentrations of fluorinated phenyl tolane isothiocyanates. The formulation based solely on the available phenyl tolane isothiocyanate compounds possesses a high melting point and extended smectic temperature range. Therefore, its results are not included here. To lower the melting point, we prepared instead a base mixture containing highly conjugated isothiocyanato tolanes, cyclohexyl tolanes, and terphenyls. The purpose is to accommodate as high an amount of phenyl tolane isothiocyanates as possible while not compromising the electro-optical performance of the final composition.

Table 2 lists the phase transition temperatures of some selected sample mixtures. As expected, higher concentration of phenyl tolane dopant brings up the melting point of the mixtures and moreover

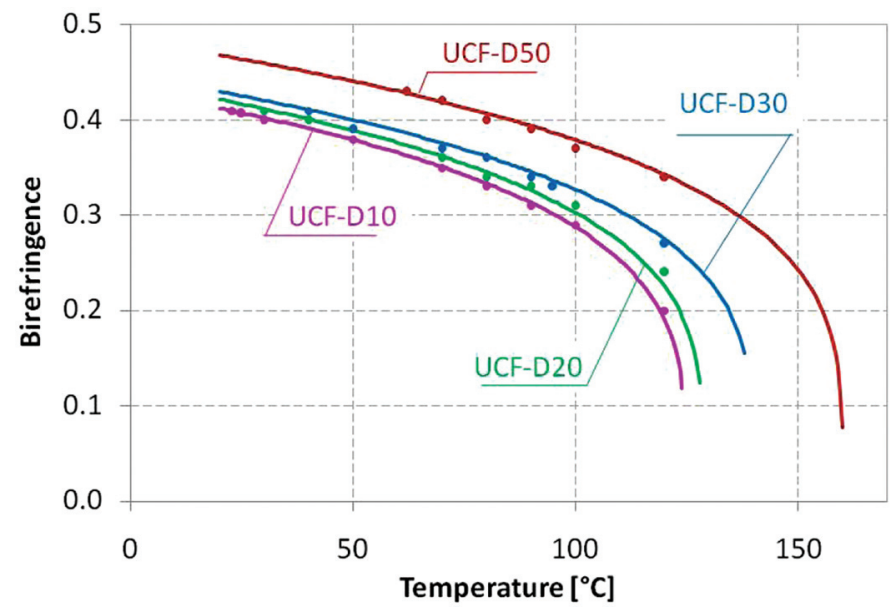

FIGURE 4 Temperature dependent birefringence of UCF-D systems. 


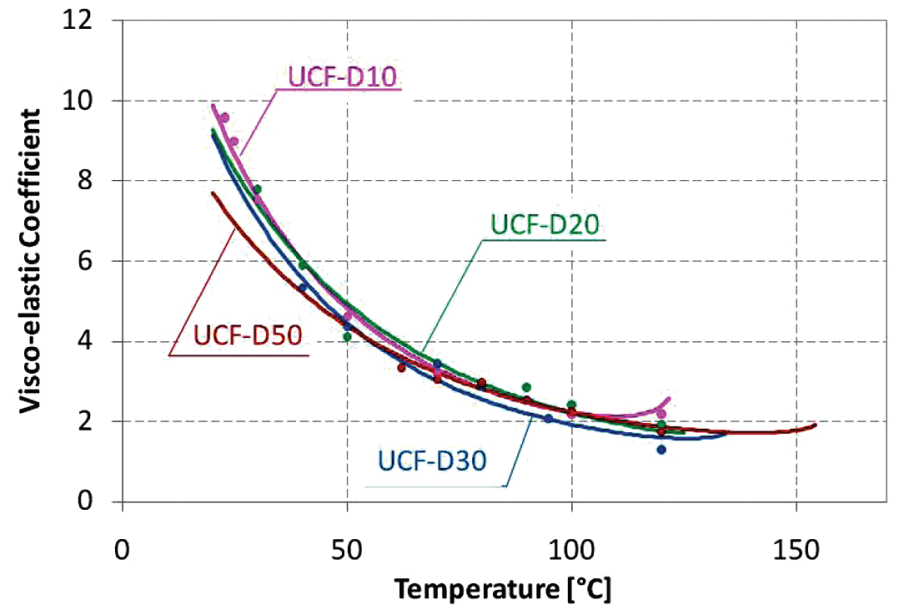

FIGURE 5 Temperature dependent visco-elastic coefficient of UCF-D systems.

the smectic to nematic transition appears. The base mixture used for our experiment shows birefringence of 0.37 . With the increased degree of dilution of the high birefringence phenyl tolanes in the base mixture, the birefringence of the UCF-Ds mixtures decreases, as shown in Figure 4. The relaxation time of the diluted mixtures is also slightly

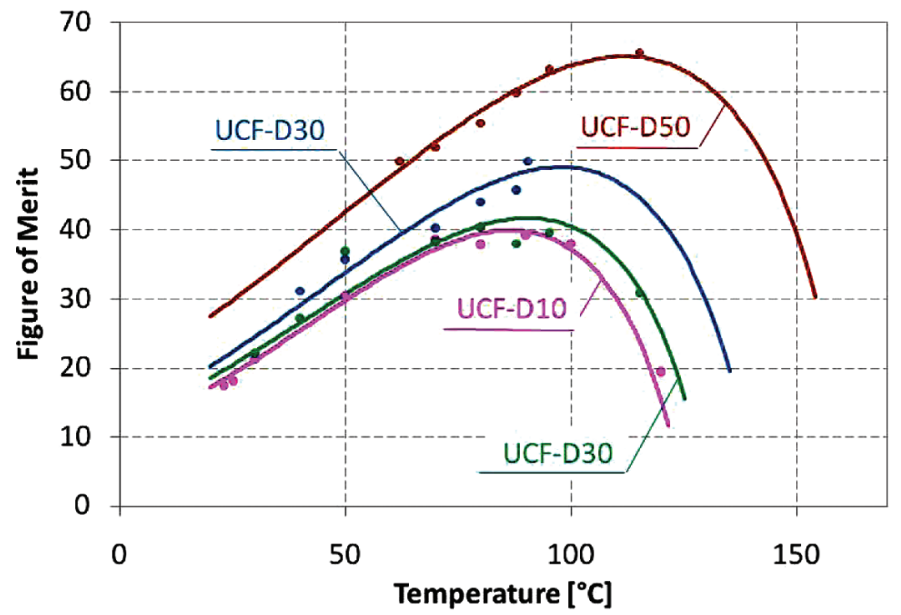

FIGURE 6 Temperature dependent FoM of UCF-D systems. 
elevated as the amount of base mixture is increased. Figure 5 compares the visco-elastic coefficient of four investigated mixtures with different degree of dilution. Figure 6 shows the temperature dependent figure-of-merit. Because of the decreased birefringence and slightly increased viscoelastic coefficient, UCF-D10 has a $30 \%$ lower FoM than UCF-D50.

\section{CONCLUSION}

We have developed eight laterally fluorinated phenyl-tolane isothiocyanates and evaluated their mesomorphic and electro-optical properties. Multiple fluorinations not only lower the melting temperature, but also increase the viscosity. A certain compromise needs to be made between high FoM and low melting temperature of the compounds. We found that difluorination of the middle phenyl group, without additional fluorination on the phenyl ring linked with terminal NCS group, results in a decrease in the visco-elastic coefficient. At present it is still unclear whether this situation is similar to the one observed for the laterally fluorinated dialkyl terphenyl compounds [35]. Further synthesis needs to be performed in order to make a more detailed comparison. The presented fluorinated phenyl-tolane isothiocyanates show high FoM and are particularly attractive for long wavelength applications, such as laser beam steering and variable optical attenuators. The UV stability of these compounds and mixtures is not a concern for IR applications. Therefore, the presented phenyl tolanes are attractive for infrared applications.

\section{REFERENCES}

[1] McManamon, P. F., Dorschner, T. A., Corkum, D. L., Friedman, L., Hobbs, D. S., Holz, M., Liberman, S., Nguyen, H. Q., Resler, D. P., Sharp, R. C., \& Watson, E. A. (1996). Proc. IEEE, 84, 268.

[2] Ren, H., Fan, Y. H., Gauza, S., \& Wu, S. T. (2004). Appl. Phys. Lett., 84, 4789.

[3] Wu, S. T. \& Yang, D. K. (2001). Reflective Liquid Crystal Displays, Wiley: New York

[4] Efron, U., Wu, S. T., Grinberg, J., \& Hess, L. D. (1985). Opt. Eng., 24, 111.

[5] Liang, X., Lu, Y. Q., Wu, Y. H., Du, F., Wang, H. Y., \& Wu, S. T. (2005). Jpn. J. Appl. Phys. 44, 1295.

[6] Nishiyama, K., Okita, M., Kawaguchi, S., Teranishi, K., \& Takamatsu, R. (2005). SID Tech. Digest, 36, 132.

[7] Lu, R., Hong, Q., Ge, Z., \& Wu, S. T. (2006). Opt. Express, 14, 6243.

[8] Harbers, G. \& Hoelen, C. (2001). SID Tech. Digest, 32, 702.

[9] Wu, S. T. \& Efron, U. (1986). Appl. Phys. Lett. 48, 624.

[10] Gauza, S., Zhu, X., Wu, S. T., Piecek, W., \& Dabrowski, U. (2007). J. Display Technology, 3, 250. 
[11] Jiao, M., Ge, Z., \& Wu, S. T. (2008). Appl. Phys. Lett. 92, 061102.

[12] Wu, S. T., Ramos, E., \& Finkenzeller, U. (1990). J. Appl. Phys. 68, 78.

[13] Wu, S. T. (1986). Phys. Rev A, 33, 1270.

[14] Wu, S. T. (1991). J. Appl. Phys., 69, 2080.

[15] Spadło, A., Dąbrowski, R., Filipowicz, M., Stolarz, Z., Przedmojski, J., Gauza, S., Fan, Y. H., \& Wu, S. T. (2003). Liq. Cryst., 30, 191.

[16] Wu, S. T. (1987). Opt. Eng, 26, 120.

[17] Wu, S. T. \& Cox, R. J. (1988). J. Appl. Phys., 64, 821.

[18] Gauza, S., Wang, H., Wen, C. H., Wu, S. T., Seed, A., \& Dabrowski, R. (2003). Jpn. J. Appl. Phys. Part 1, 42, 3463.

[19] Wu, S. T., Margerum, J. D., Meng, B., Dalton, L. R., Hsu, C. S., \& Lung, S. H. (1992). Appl. Phys. Lett., 61, 630.

[20] Wu, S. T., Neubert, M., Keast, S. S., Abdallah, D. G., Lee, S. N., Walsh, M. E., \& Dorschner, T. A. (2000). Appl. Phys. Lett. 77, 957.

[21] Wu, S. T., Hsu, C. S., \& Shyu, K. F. (1999). Appl. Phys. Lett., 74, 344.

[22] Wu, S. T., Hsu, C. S., Chuang, Y. Y., \& Cheng, H. B. (2000). Jpn. J. Appl. Phys. Part 2, 39, L38.

[23] Wu, S. T., Margerum, J. D., Ho, M. S., Fung, B. M., Hsu, C. S., Chen, S. M., \& Tsai, K. T. (1995). Mol. Cryst. Liq. Cryst. Sci. Technol. Sec. A , 261, 79.

[24] Seed. A. J., Toyne, K. J., Goodby, J. W., \& Hird, M. (2000). J. Mater. Chem., 10, 2069.

[25] Sekine, C., Konya, N., Minai, M., \& Fujisawa, K. (2001). Liq. Cryst. 28, 1361.

[26] Sekine, C., Ishitobi, M., Iwakura, K., Minai, M., \& Fujisawa. K. (2002). Liq. Cryst., 29, 355.

[27] Sekine, C., Konya, N., Minai, M., \& Fujisawa, K. (2001). Liq. Cryst., 28, 1495.

[28] Catanescu, O. \& Chien, L. C. (2006) Liq. Cryst., 33, 115.

[29] Gauza. S., Wu, S. T., Spadlo, A., \& Dabrowski, R. (2006). J. Display Technology, $2,247$.

[30] Gauza, S., Wen, C. H., Wu, B., Wu, S. T., Spadlo, A., \& Dabrowski, R. (2006). Liq. Cryst., 33, 705.

[31] Gauza, S., Wen, C. H., Wu, S. T., Janarthanan, N., \& Hsu, C. S. (2004). Jpn. J. Appl. Phys., 43, 7634.

[32] Wu, S. T., Efron, U., \& Hess, L. D. (1984). Appl. Opt., 23, 3911.

[33] Wu, S. T., Lackner, A. M., \& Efron, U. (1987). Appl. Opt., 26, 3441.

[34] Wu, S. T. \& Wu, C. S. (1990). Phys. Rev. A., 42, 2219.

[35] Pauluth, D. \& Tarumi, K. (2004). J. Materials Chemistry, 14, 1219. 